\title{
From the uterus to 12 months of age: changes in the maternal-child health profile in three birth cohorts in Pelotas, Rio Grande do Sul State, Brazil, 1982-2004
}

This special supplement of Cadernos de Saúde Pública/Reports in Public Health presents the comparative results of three birth cohorts followed in Pelotas, Rio Grande do Sul, Brazil, in relation to the children's first 12 months of life. The analyses included more than 15 thousand births from 1982, 1993, and 2004, a period in which Brazil experienced profound political, social, and economic transformations. Accompanying this process, during these 22 years the country has witnessed important changes in the epidemiological profile of health problems and risk exposures, as well as a total reorganization of the national health system, with the creation of the Unified National Health System in 1988. The results presented in this supplement paint a faithful portrait of these changes, viewed from the perspective of a medium-sized city in the southern region of the country. However, the uniqueness of the data source does not limit its validity for representing a more general reality - the comparison of some results from the Pelotas cohorts to national averages shows great similarities. Meanwhile, the quality of information from cohort studies, preserving the temporality of the events, gives special importance to the panorama presented here. The fact that not just one but three birth cohorts have been performed in the same geographic base, and at 11-year intervals, places Brazil in a prominend position in the international epidemiological scenario. This is a unique situation among developing countries. Some wealthy countries like the United States and France, although experienced in conducting adult cohorts, are just now launching their own birth cohorts. The advantages of this type of study include the possibility of testing hypotheses on long-term effects of exposures occurring during pregnancy (through fetal programming) and in early childhood on morbidity and causes of mortality in adulthood. At a time when complex chronic non-communicable diseases dominate the morbidity profile of both wealthy countries and poor countries undergoing demographic and nutritional transition, the early identification of individuals at risk of becoming ill assumes a vital role. In addition, cohorts allow a detailed follow-up of the life cycle and effect of life histories (for example, gaining/losing weight; getting richer/poorer; and vice versa) on this cycle.

The Pelotas cohorts have been visited regularly, and the oldest of the three, launched in 1982, has already undergone more than a dozen follow-ups (including either the entire cohort or sub-samples). The youngest cohort, from 2004, is already in its fifth follow-up at four years of age. Follow-up of the three cohorts when the study participants reach the same chronological age has allowed documenting the "epidemiological transition" in various health aspects. As appears clearly in the following articles, during these 22 years important changes were observed in relation to economic situation, birth weight, prematurity, mortality, and nutritional status, among others. We urge readers to examine this information in the articles, in the hope that it can be used to back action, programs, and policies. At the local level, based on the results for infant mortality, the Pelotas Municipal Health Secretariat has requested support for planning and implementing interventions to improve the infant health situation. A series of measures were implemented, resulting in a reduction in infant mortality from 20 to 12.3 per thousand live births from 2004 to 2007, thus equaling the mean value for the State of Rio Grande do Sul as a whole.

\section{Iná S. Santos}

Programa de Pós-graduação em Epidemiologia, Universidade Federal de Pelotas, Pelotas, Brasil.

inasantos@uol.com.br
Aluísio J. D. Barros

Programa de Pós-graduação em Epidemiologia, Universidade Federal de Pelotas, Pelotas, Brasil.

abarros.epi@gmail.com 